\title{
Coincidence detection of inharmonic pulses in a nonlinear crystal
}

\author{
Xavier Vidal, ${ }^{1,2}$ Pablo Balenzuela, ${ }^{3, *}$ Javier M. Buldú, ${ }^{4, \dagger}$ Jordi Martorell, ${ }^{1,2}$ and Jordi García-Ojalvo ${ }^{2}$ \\ ${ }^{1}$ ICFO-Institut de Ciencies Fotoniques, Mediterranian Technology Park, 08860 Castelldefels, Spain \\ ${ }^{2}$ Departament de Física i Enginyeria Nuclear, Universitat Politècnica de Catalunya, Colom 11, E-08222 Terrassa, Spain \\ ${ }^{3}$ Departamento de Física, Facultad de Ciencias Exactas y Naturales, Universidad de Buenos Aires, Pabellón 1, \\ Ciudad Universitaria (1428), Buenos Aires, Argentina \\ ${ }^{4}$ Departamento de Física, Universidad Rey Juan Carlos, Tulipán s/n, 28933 Móstoles, Spain
}

(Received 27 July 2006; revised manuscript received 10 October 2006; published 23 January 2007)

\begin{abstract}
Two trains of light pulses at periods that are equally shifted from the harmonics of a missing fundamental are combined in a nonlinear crystal. As a result of a noncollinear phase-matched second-order nonlinear generation, a new train of pulses is obtained. When the temporal width of the input pulses is large, the frequency of the resulting pulse train follows the observations from classical experiments on the perception of virtual pitch by the brain. On the other hand, when the width of the input pulses is small, the generated pulse train exhibits much lower frequencies, analogous to those observed in the motor neural system. Our experimental setup allows us to explore, systematically and continuously, the transition between these two regimes, while at the same time to demonstrate that the functionalities that have been observed in the nervous system are similar to the ones we observe from coincidence detection in quadratic nonlinear systems.
\end{abstract}

DOI: 10.1103/PhysRevE.75.012902

PACS number(s): 43.64.+r, 05.45.-a, 42.65.Sf

One of the most remarkable features of nonlinear systems is their ability to process complex input signals. A classical example is the perception of virtual pitch by the brain. In that context, it is well known [1] that a missing fundamental tone can be perceived upon exposure to only some of its harmonics. Psycophysical experiments by Schouten et al. [2] showed that when a set of consecutive harmonics were equally shifted in frequency, it was not the frequency difference (i.e., the original fundamental) that was perceived. Instead, the perceived pitch varied linearly with the frequency shift. Specifically, Schouten and co-workers considered input tones of frequencies

$$
f_{i}=(k+i-1) f_{0}+\Delta f, \quad i=1, \ldots, n,
$$

where $f_{0}$ is the missing fundamental frequency, $k>1$ is an integer, and $\Delta f$ is the frequency detuning which makes the input frequencies to be inharmonic. Under these conditions, the perceived pitch was seen to be given by a frequency that matches the following expression:

$$
f_{r}=f_{0}+\frac{\Delta f}{k+(n-1) / 2} .
$$

Recently, Chialvo et al. [3] proposed a simple and elegant mechanism that accounts for this response, involving a linear superposition of the input harmonics and a nonlinear noisy detection of the frequency (2) via a threshold. The mechanism, subsequently named ghost resonance, has been experimentally verified in lasers [4-6] and in electronic circuits [7].

Experimental results using magnetoencephalographic measurements [8] showed that the missing fundamental illusion also arises when the harmonic inputs are presented

\footnotetext{
*Electronic address: balen@df.uba.ar

${ }^{\dagger}$ Electronic address: javier.buldu@urjc.es
}

binaurally-i.e., when different harmonics are applied to each of the two ears. The mechanism of Chialvo et al. [3] was extended to that situation by modeling separate neuronal pathways that detected two different input harmonics [9]. That study showed that in the context of distributed inputs, the mechanism of ghost resonance heavily relies on the coincidence detection of synaptic pulse trains (transduced by the input neurons that receive the input harmonic signals) by an integrating neuron. An experimental realization of this effect has been recently performed in a real neurophysiological setting involving the motor system of cats [10]. That experiment has shown that when the input trains are inharmonic (i.e., frequency shifted with respect to the original harmonics), the processing neuronal pool responds at frequencies much lower that those expected from expression (2). Subsequent experiments with nonlinear electronic circuits [11] indicate that the difference in the response is due to the small width of the pulses acting upon the integrating neuron, in contrast with the larger width presumably associated with acoustic neuronal pathways.

The examples of ghost resonance studied so far, and described above, involved systems exhibiting excitability, a property shared by neurons and many other physical systems, characterized by the existence of an activation threshold. Even though the original mechanism proposed by Chialvo et al. [3] relied on the existence of such a threshold, recent studies [9-11] have shown that the main ingredient of the phenomenon, when the inputs are distributed upon different coupled-threshold devices, is coincidence detection. In this paper, we use an experimental setup that does not exhibit excitability, but that provides a mechanism of coincidence detection. Specifically, we use the phenomenon of secondharmonic generation in a nonlinear optical crystal, through which light at a given optical frequency $\left(2 f_{\text {opt }}\right)$ is generated within the crystal only when two distinct beams at half that optical frequency $\left(f_{\text {opt }}\right)$ are present. We show that ghost resonance arises also in this context and address the question of 


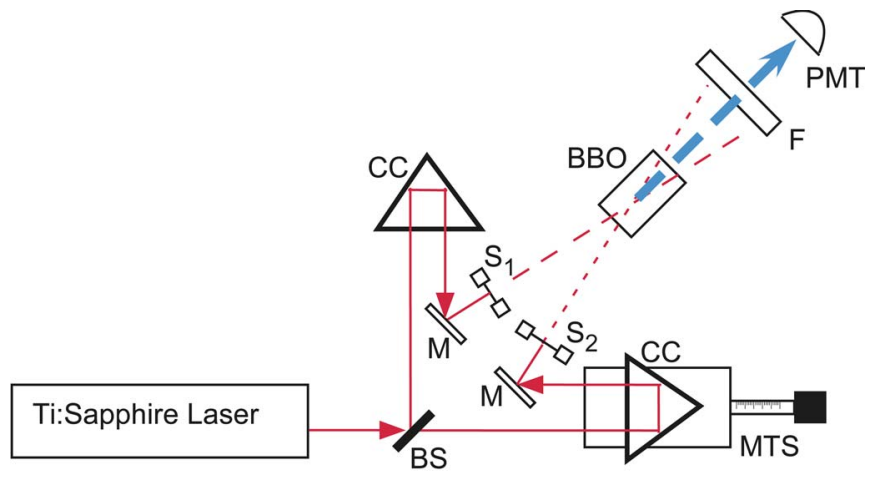

FIG. 1. (Color online) Schematic representation of the experimental array. We split the laser output, thin line, into two beams with a beam splitter, BS, and determine the frequencies of the pulses using one shutter for each beam, $S 1$ and $S 2$, both of them controlled by a code written in LABVIEW (National Instruments). The thick dashed line is for the second harmonic at the exit of the nonlinear crystal, BBO; $M$ are mirrors, $F$ are filters to stop the pump beam, CC are corner cubes, PMT is a photomultiplier tube, and MTS indicates micrometric translation stage.

the influence of the input pulse width on the ghost resonance response. This allows us to (i) perform systematic measurements for continuously varying pulse widths, with high controllability and reproducibility, (ii) ascertain the generality of the phenomenon reported, which is seen to arise in any system that operates via coincidence detection, and (iii) suggest a possible functional role of this phenomenon in nonlinear photonic devices, with potential applications in all-optical signal processing. Lasers have already been proposed, in the context of optical neurocomputing, as building blocks in optical neural networks [12], which perform functions such as routing, buffering, and associative memory [13]. The results presented here indicate that similar functionalities might be expected from simpler nonlinear optical elements.

The experimental setup is shown in Fig. 1. A 76-MHz train of 130-fs pulses, produced by a Ti-sapphire laser at a wavelength of $800 \mathrm{~nm}$, was divided into two beams using a $50 / 50$ beam splitter. These two beams are recombined in a BBO crystal cut for noncollinear phase matching, which generates second-harmonic light at a wavelength of $400 \mathrm{~nm}$ when both beams are present simultaneously in the crystal. The coincidence of the short laser pulses is obtained using a movable translation stage, as shown in Fig. 1. Both beams are gated using electromechanical shutters $S_{1}$ and $S_{2}$ at respective frequencies $f_{1}$ and $f_{2}$ on the order of $\mathrm{Hz}$, much smaller than the repetition rate of the pulsed laser beam. This leads to wide light pulses which are, in turn, composed of the 130-fs ultrashort pulses described above. In what follows, we will refer to those wide gated packages as pulses, ignoring their substructure at ultrashort time scales, which is not relevant for the behavior reported here.

The gating process is computerized, which enables control of the shutter frequencies and ensures a constant (stable) phase relation between them. The input signals consist of two pulse trains of dynamical frequencies $f_{1}$ and $f_{2}$, represented by the two upper thin traces in Fig. 2. The width and dynamical frequency of the pulses are controlled by the optical shutters.

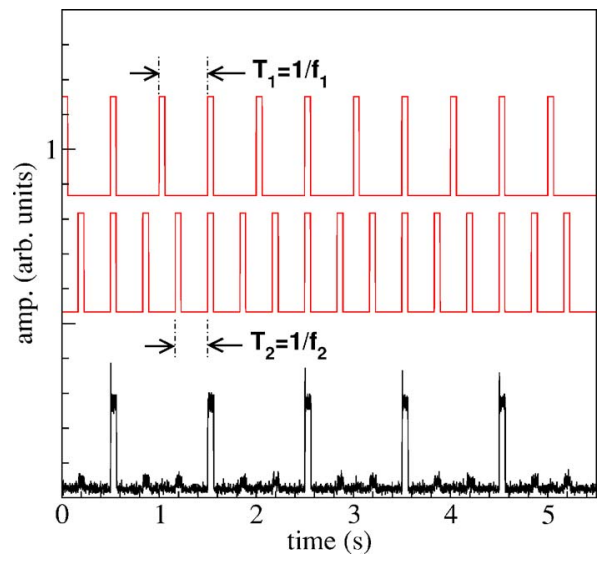

FIG. 2. (Color online) Time series of the input pulsed signals (two upper lines, shifted vertically for clarity) of frequencies $2 \mathrm{~Hz}$ and $3 \mathrm{~Hz}$ and of the response signal of the nonlinear crystal (lower line) at the ghost frequency $1 \mathrm{~Hz}$, as detected by the photomultiplier. The amplitude of the input signals has been vertically shifted in order to ease comparison between time series.

When two input pulses coincide inside the nonlinear crystal (upper two time traces in Fig. 2), the generated light at $2 f_{\text {opt }}$ is detected by the photomultiplier (lower time trace in Fig. 2). The nonlinear crystal acts as a coincidence detector and replaces the threshold of detection of previous systems where ghost resonance has been studied $[3,7,9,11]$. In the particular case of Fig. 2, we have set $f_{1}=2 \mathrm{~Hz}$ and $f_{2}=3 \mathrm{~Hz}$, which corresponds to $n=2, k=2, f_{0}=1 \mathrm{~Hz}$, and $\Delta f=0$ in Eqs. (1) and (2). Given the thresholdless nature of the quadratic nonlinear interaction, in this particular case a pulse train at the ghost frequency $\left(f_{r}=1 \mathrm{~Hz}\right.$ [see Eq. (2)]) is always generated and its detection is only limited by the sensibility of the overall detection system.

In order to check the validity of Eq. (2) for inharmonic inputs, we now detune $f_{1}$ away from the harmonic of $f_{0}$ by setting $f_{1}=k f_{0}+\Delta f$, varying $\Delta f$ between 0 and $1 \mathrm{~Hz}$ while keeping $f_{2}=f_{1}+f_{0}$ and $f_{0}$ fixed. We start by setting the pulse width $\Delta t_{p}$ to a relatively large value: namely, $\Delta t_{p}=60 \mathrm{~ms}$. Figure 3(a) shows the instantaneous response frequency $f_{r}$ (defined as the inverse of the time interval between output pulses) as a function of the input frequency $f_{1}$. The response frequency is seen to follow well the relation predicted by Eq. (2) for $n=2$ and $k=2,3$ (dashed lines in the figure) for almost the whole range of $f_{1}$. Note that for a fixed $f_{0}$, a given value of $f_{1}$ can be obtained from Eq. (1) via different choices of $k$ and $\Delta f$. That is the reason why multiples lines of different $k$ are observed in Fig. 3(a) for the same range of $f_{1}$ values.

A linear response at large frequencies is also observed, however, in plot 3(a). This high-frequency response is a direct consequence of consecutive coincidences of the (wide) pulses, as can be seen from Fig. 4. The resulting frequency is the inverse of the silent period of the slower input signal $\left(T_{1}=\frac{1}{f_{1}}-\Delta t_{p}\right)$, as can be deduced from this figure, and therefore, for this case, the response frequency $f_{r}$ should follow the curve 

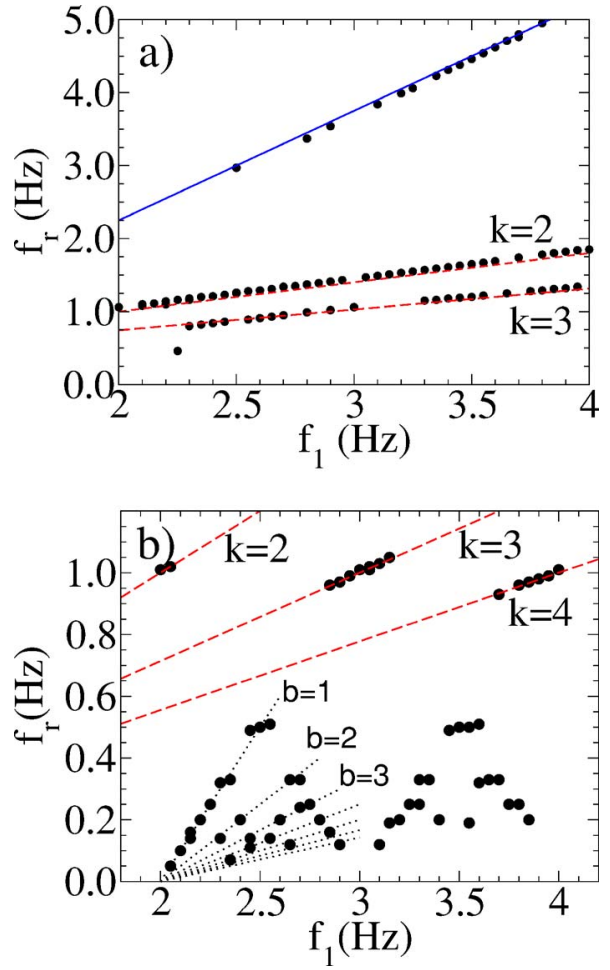

FIG. 3. (Color online) Interpulse instantaneous frequency for increasing values of $\Delta f$. The horizontal axis corresponds to the slow input frequency $f_{1}$ given by $f_{1}=k f_{0}+\Delta f$. The shutters were here driven for trains of rectangular pulses of width $60 \mathrm{~ms}$ (a) and $5 \mathrm{~ms}$ (b). Symbols (black circles) represent experimental data that are statistically significant (representing more than $10 \%$ of all interpulse events); the dashed lines labeled $k=2,3,4$ (in both panels) indicate the expected ghost frequencies predicted by Eq. (2), and the unlabeled solid line in panel (a) represents the relation given in Eq. (3). Finally, the dotted lines labeled $b=1,2,3$ in (b) represent the low-frequency response at $f_{r}=\Delta f / b$ (see text).

$$
f_{r}=\frac{1}{1 / f_{1}-\Delta t_{p}} .
$$

This expression is represented by the upper (unlabeled) solid line in Fig. 3(a) and exhibits good agreement with the experimental results.

We now turn our attention to the opposite limit of very narrow pulses. To that end we apply pulses of width $\Delta t_{p}=5 \mathrm{~ms}$ (the narrowest we can get given the speed limitations of the shutters). Figure 3(b) shows the instantaneous response frequency $f_{r}$, again as a function of slowest input frequency $f_{1}$. We can see that the relation given by Eq. (2) holds only in the neighborhood of the harmonic case $\left(f_{1}=2 \mathrm{~Hz}\right.$ and $\left.3 \mathrm{~Hz}, f_{r}=1 \mathrm{~Hz}\right)$. On the other hand, in most of the inharmonic region, the system responds with pulse trains of very low frequencies, in agreement with previous experimental results in the motor neural system [10] and in electronic circuits [11]. These responses are grouped in families of lines following $f_{r}=\frac{\Delta f}{b}$, with $b$ being an integer, as shown in Fig. 3(b). The line with $b=1$ was observed experimentally in Ref. [11], and its origin was determined analytically. The closer we are to the limit of zero-width pulses, the more lines

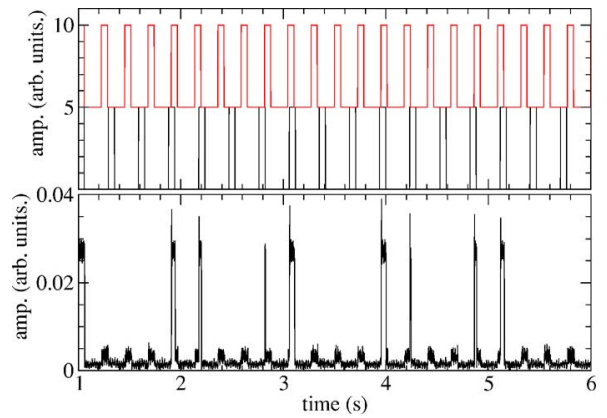

FIG. 4. (Color online) Time series of the input signals (upper panel, shifted vertically for clarity) and response signal of the nonlinear crystal (lower panel), in the inharmonic case, for frequencies $f_{1}=3.4 \mathrm{~Hz}$ and $f_{2}=4.4 \mathrm{~Hz}$. We can see two dominant frequencies in this response: the lowest frequency corresponds to the values predicted by Eq. (2), and the higher one arises from consecutive coincidences of the broad pulses used in these sequence of experiments. Here the pulse width was set to $\Delta t_{p}=60 \mathrm{~ms}$.

appear. These lines arise from the condition of coincidence,

$$
l T_{1}=m T_{2},
$$

where $l$ and $m$ are integers and $T_{1}$ and $T_{2}$ are the input periods: respectively, $T_{1}=\frac{1}{f_{1}}=\frac{1}{k f_{0}+\Delta f}$ and $T_{2}=\frac{1}{f_{2}}=1 /(k+1) f_{0}+\Delta f$. When the condition given by Eq. (4) is fulfilled, both input trains coincide and a pulse is detected. When potential coincidences at frequency $\Delta f \neq 0$ are themselves missed, lower frequencies $\frac{\Delta f}{b}$ are generated. This gives rise to different families of lines [quasipyramids in Fig. 3(b)], depending on the value of $k$ that relates $f_{1}$ and $f_{0}$.

The two situations depicted in plots (a) and (b) of Fig. 3 represent two opposite limits of the coincidence detection mechanism: for long pulse widths the standard response of the system is given by Eq. (2) [dashed lines labeled $k=2,3$ in Fig. 3(a)], whereas for short pulse widths the system responds preferentially at much smaller frequencies of the order of $\Delta f$ [dotted lines labeled $b=1,2,3$ in Fig. 3(a)]. In order to systematically investigate the transition between these two scenarios, we now fix the frequency shift $\Delta f \neq 0$ and vary continuously the pulse width. The corresponding behavior is shown in Fig. 5. In this figure, horizontal dashed lines represent the standard ghost frequency responses as predicted from Eq. (2). We are interested in examining how the response at low frequencies (data points at frequencies below the $k=3$ line in the figure) behave as the width of the input pulses increase. Figure 5 shows how the low frequencies gradually increase with the width of the input pulses, until only the standard ghost responses remain for pulse widths larger than $40 \mathrm{~ms}$.

We have used here a classical nonlinear optical interaction as a tool to investigate the processing of complex distributed signals via the paradigm of ghost resonance. This phenomenon provides the simplest explanation to date of the longstanding problem of the missing fundamental illusion. Our results show that excitability, ubiquitous, for instance, in neural systems (where the missing fundamental illusion was originally reported), is not necessary for ghost resonance to 


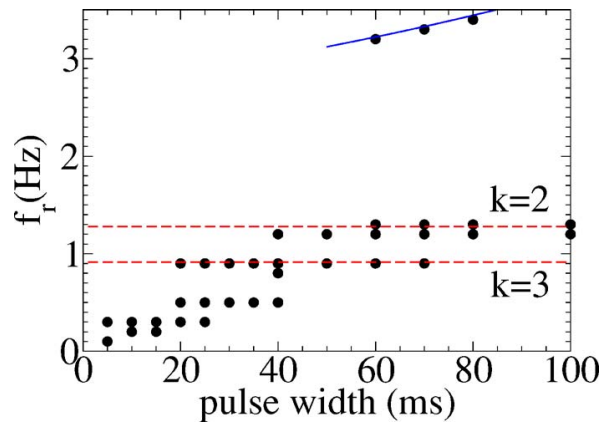

FIG. 5. (Color online) Interpulse instantaneous frequency for increasing values of $\Delta_{f}$ as a function of the pulse width. The input frequencies are $f_{1}=2.7 \mathrm{~Hz}$ and $f_{2}=3.7 \mathrm{~Hz}$, which correspond to a detuning $\Delta f=0.7 \mathrm{~Hz}$ with respect to the harmonic $k=2$ frequencies $2.0 \mathrm{~Hz}$ and $3.0 \mathrm{~Hz}$ [see Eq. (2)]. As in Fig. 3, symbols (black circles) represent significant experimental data; the dashed lines labeled $k=2,3$ indicate the expected response frequencies predicted by Eq. (2). The solid unlabeled line represents the relation given in Eq. (3).

occur. A system that is not excitable but only shows coincidence detection, such as the one presented here, exhibits the same phenomenology. We use second-harmonic generation as a means of separating the input signals from the output response, the same thing that a neuron does when transducing the applied input currents into a membrane potential.

The results clearly show a transition from a lowfrequency response regime to another regime completely dominated by relation (2), as the width of the pulses increases. Such a transition between regimes could underlie the differences observed between previous psychophysical experiments on auditory response [2] and recent experiments on the motor neural system [10]. In the former, expression (2) held unambiguously; in the latter, on the other hand, that behavior gave way very frequently to low-frequency responses. Correspondingly, synaptic pulses are known to be wider in the auditory system than in the motor reflex system. Finally, from a technological viewpoint, the present results also show that nonlinear optical crystals carry out nontrivial signal processing tasks that mimic those of more complicated systems such as the brain.

We thank Claudio Mirasso and Elías Manjárrez for fruitful discussions. Financial support was provided by MCyTFEDER (Spain, Projects Nos. BFM2003-07850, TEC200507799, MAT2005-06354, FIS2006-11452), by the ECfunded project PHOREMOST (FP6/2003/IST/2511616), and by the Generalitat de Catalunya. P.B. is a member of "Carrera de Conicet," Argentina.
[1] H. von Helmholtz, On the Sensations of Tone as a Physiological Basis for the Theory of Music (Kessinger, Whitefish, Montana, 2005) (republication of the original 1885 edition).

[2] J. F. Schouten, R. J. Ritsma, and B. L. Cardozo, J. Acoust. Soc. Am. 34, 1418 (1962).

[3] D. R. Chialvo, O. Calvo, D. L. Gonzalez, O. Piro, and G. V. Savino, Phys. Rev. E 65, 050902(R) (2002).

[4] J. M. Buldú, D. R. Chialvo, C. R. Mirasso, M. C. Torrent, and J. García-Ojalvo, Europhys. Lett. 64, 178 (2003).

[5] J. M. Buldú, C. M. Gonzalez, J. Trull, J. García-Ojalvo, and M. C. Torrent, Chaos 15, 013103 (2005).

[6] G. Van der Sande, G. Verschaffelt, J. Danckaert, and C. R. Mirasso, Phys. Rev. E 72, 016113 (2005).
[7] O. Calvo and D. R. Chialvo, Int. J. Bifurcation Chaos Appl. Sci. Eng. 16, 731 (2006).

[8] C. Pantev, T. Elbert, B. Ross, C. Eulitz, and E. Terhardt, Hear. Res. 100, 164 (1996).

[9] P. Balenzuela and J. García-Ojalvo, Chaos 15, 023903 (2005).

[10] E. Manjárrez, P. Balenzuela, J. García-Ojalvo, L. Martínez, A. Flores, and C. R. Mirasso, Biosystems (to be published).

[11] A. Lopera, J. M. Buldú, M. C. Torrent, D. R. Chialvo, and J. García-Ojalvo, Phys. Rev. E 73, 021101 (2006).

[12] E. C. Mos, J. Schleipen, H. de Waardt, and G.-D. Khoe, IEEE J. Quantum Electron. 36, 486 (2000).

[13] F. C. Hoppensteadt and E. M. Izhikevich, Phys. Rev. E 62, 4010 (2000). 